\title{
Derivative-Free Superiorization: Principle and Algorithm
}

\author{
Yair Censor • Edgar Garduño • \\ Elias S. Helou • Gabor T. Herman
}

Received: date / Accepted: date / Revised: October 21, 2020

\begin{abstract}
The superiorization methodology is intended to work with input data of constrained minimization problems, that is, a target function and a set of constraints. However, it is based on an antipodal way of thinking to what leads to constrained minimization methods. Instead of adapting unconstrained minimization algorithms to handling constraints, it adapts feasibility-seeking algorithms to reduce (not necessarily minimize) target function values. This is done by inserting target-function-reducing perturbations into a feasibility-seeking algorithm while retaining its feasibility-seeking ability and without paying a high computational price. A superiorized algorithm that employs component-wise target function reduction steps is presented. This enables derivative-free superiorization (DFS), meaning that superiorization can be applied to target functions that have no calculable partial derivatives or subgradients. The numerical behavior of our derivative-free superiorization algorithm is illustrated on a data set generated by simulating a problem of image reconstruction from projections. We present a tool (we call it a proximity-target curve) for deciding which of two iterative methods is "better" for solving a particular problem. The plots of proximity-target
\end{abstract}

Edgar Garduño would like to thank the support of DGAPA-UNAM. The work of Yair Censor is supported by the ISF-NSFC joint research program Grant No. 2874/19. Elias S. Helou was partially supported by CNPq grant No. 310893/2019-4.

Yair Censor

Department of Mathematics, University of Haifa, Mt. Carmel, Haifa 3498838, Israel

E-mail: yair@math.haifa.ac.il

Edgar Garduño

Departamento de Ciencias de la Computación, Instituto de Investigaciones en Matemáticas Aplicadas y en Sistemas, Universidad Nacional Autónoma de México, Cd. Universitaria, C.P. 04510, Mexico City, Mexico

E-mail: edgargar@ieee.org

Elias S. Helou

Instituto de Ciências Matemáticas e de Computação, Universidade of São Paulo, São Carlos, São Paulo 13566-590, Brazil

E-mail: elias@icmc.usp.br

Gabor T. Herman

Computer Science Ph.D. Program, The Graduate Center, City University of New York, New York, NY 10016, USA

E-mail: gabortherman@yahoo.com 
curves of our experiments demonstrate the advantage of the proposed derivative-free superiorization algorithm.

Keywords derivative-free $\cdot$ superiorization $\cdot$ constrained minimization $\cdot$ componentwise perturbations · proximity function $\cdot$ bounded perturbations $\cdot$ regularization

Mathematics Subject Classification (2010) 65K05 $65 \mathrm{~K} 15 \cdot 90 \mathrm{C} 56$

\section{Introduction}

1.1 The superiorization methodology (SM)

In many applications there exist efficient iterative algorithms for producing constraintscompatible solutions. Often these algorithms are perturbation resilient in the sense that, even if certain kinds of changes are made at the end of each iterative step, the algorithms still produce a constraints-compatible solution. This property is exploited in superiorization by using such perturbations to steer an algorithm to an output that is as constraints-compatible as the output of the original algorithm, but is superior (not necessarily optimal) to it with respect to a given target function.

Superiorization has a world-view that is quite different from that of classical constrained optimization. Both in superiorization and in classical constrained optimization there is an assumed domain $\Omega$ and a criterion that is specified by a target function $\phi$ that maps $\Omega$ into $\mathbb{R}$. In classical optimization it is assumed that there is a constraints set $C$ and the task is to find an $\boldsymbol{x} \in C$ for which $\phi(\boldsymbol{x})$ is minimal over $C$. Two difficulties with this approach are: (1) The constraints that arise in a practical problem may not be consistent, so $C$ could be empty and the optimization task as stated would not have a solution. (2) Even for nonempty $C$, iterative methods of classical constrained optimization typically converge to a solution only in the limit and some stopping rule is applied to terminate the process. The actual output at that time may not be in $C$ (especially if the iterative algorithm is initialized at a point outside $C$ ) and, even if it is in $C$, it is most unlikely to be a minimizer of $\phi$ over $C$.

Both issues are handled in the superiorization approach investigated here by replacing the constraints set $C$ by a nonnegative real-valued proximity function $\mathcal{P} r_{T}$ that indicates how incompatible a given $\boldsymbol{x} \in \Omega$ is with specified constraints $T$. Then the merit of an actual output $\boldsymbol{x}$ of an algorithm is represented by the smallness of the two numbers $\mathcal{P} r_{T}(\boldsymbol{x})$ and $\phi(\boldsymbol{x})$. Roughly, if an iterative algorithm produces an output $\boldsymbol{x}$, then its superiorized version will produce an output $\boldsymbol{x}^{\prime}$ for which $\mathcal{P} r_{T}\left(\boldsymbol{x}^{\prime}\right)$ is not larger than $\mathcal{P r}_{T}(\boldsymbol{x})$, but (as in-practice demonstrated) generally $\phi\left(\boldsymbol{x}^{\prime}\right)$ is smaller than $\phi(\boldsymbol{x})$.

As an example, let $\Omega=\mathbb{R}^{J}$ and consider a set $T$ of constraints of the form

$$
\left\langle\boldsymbol{d}^{i}, \boldsymbol{x}\right\rangle=h_{i}, \quad i=1,2, \ldots, I,
$$

where $\boldsymbol{d}^{i} \in \mathbb{R}^{J}$ and $h_{i} \in \mathbb{R}$, for all $i=1,2, \ldots, I$, and $\langle\cdot, \cdot\rangle$ is the Euclidean inner product in $\mathbb{R}^{J}$. There may or may not be an $\boldsymbol{x} \in \mathbb{R}^{J}$ that satisfies this set of constraints, but we can always define a proximity function for $T$ as, for example, by

$$
\mathcal{P} r_{T}(\boldsymbol{x}):=\sum_{i=1}^{I}\left(\left\langle\boldsymbol{d}^{i}, \boldsymbol{x}\right\rangle-h_{i}\right)^{2}
$$


There are several approaches in the literature that attempt to minimize both competing objectives $\mathcal{P} r_{T}(\boldsymbol{x})$ and $\phi(\boldsymbol{x})$ as a way to handle constrained minimization. The oldest one is the penalty function approach, also useful in regularization of inverse problems [19]. In that approach, the constrained minimization problem is replaced by the unconstrained minimization of the combination $\phi(\boldsymbol{x})+\pi \mathcal{P} r_{T}(\boldsymbol{x})$, in which $\pi \geq 0$ is a penalty parameter that governs the relative importance of minimizing the two summands. An inherent difficulty with this is that the penalty parameter needs to be chosen by the user. The filter method approach [20], among others, was developed to avoid this difficulty. Of course, people have also applied multiobjective minimization with two objectives (bi-objective minimization) to the competing objectives $\mathcal{P} r_{T}(\boldsymbol{x})$ and $\phi(\boldsymbol{x})$. None of these approaches are close in their underlying principles to the superiorization methodology employed in this paper.

1.2 Derivative-free superiorization: Expanding the boundaries of superiorization and competing with derivative-free optimization

Our motivating purpose in this paper is to investigate the general applicability of derivative-free superiorization (DFS) as an alternative to previously proposed superiorization approaches. These earlier approaches were based on generation of nonascending vectors, for target function reduction steps, that mostly required the ability to calculate gradients or subgradients of the target function. Observing the body of knowledge of derivative-free optimization (DFO), see, e.g., [14], we explore a DFS algorithm and demonstrate its action numerically.

In the perturbation phase of the superiorized version of a basic algorithm we replace the target function reduction steps that depend on gradient or subgradient calculations by steps that use a direction search technique which does not require any form of differentiability. Continuing the work of [10], we search the neighborhood of a current point $\boldsymbol{x}$ for a point at which the target function exhibits nonascent. A specific scheme for doing this is described in detail below; particularly, in Section 3.

While this might seem a simple technical matter, the ramifications for practical applications of the SM are important. For example, in intensity-modulated radiation therapy treatment planning, with photons, protons or other particles, the normal tissue complication probability (NTCP) is a predictor of radiobiological effects for organs at risk. The inclusion of it, or of other biological functions, as an objective function in the mathematical problem modeling and the planning algorithm, is hampered because they are, in general, empirical functions whose derivatives cannot be calculated, see, e.g., [23]. In the recent paper [37] the authors list issues of immediate clinical and practical relevance to the Proton Therapy community, highlighting the needs for the near future but also in a longer perspective. They say that "...practical tools to handle the variable biological efficiency in Proton Therapy are urgently demanded...".

The output of a superiorized version of a constraints-compatibility-seeking algorithm will have smaller (but not minimal) target function $\phi$ value than the output by the same constraints-compatibility-seeking algorithm without perturbations, everything else being equal. Even though superiorization is not an exact minimization method, we think of it as an applicable (and possibly, more efficacious) alternative to derivative-free constrained minimization methods applied to the same data for two main reasons: its ability to handle constraints and its ability to cope with very large-size problems. This is in contrast with the current state of the art, which is as follows. 
The review paper of Rios and Sahinidis [40] "... addresses the solution of boundconstrained optimization problems using algorithms that require only the availability of objective function values but no derivative information," with bound constraints imposed on the vector $\boldsymbol{x}$. The book by Conn, Scheinberg and Vicente [14] deals only with derivative-free unconstrained minimization, except for its last chapter (of 10 pages out of the 275) entitled "Review of constrained and other extensions to derivative-free optimization." Li et al. [32] do not even mention constraints. In [17] the numerical work deals with: "The dimension of the problems [i.e., the size of the vector $\boldsymbol{x}$ ] varies between 2 and 16, while the number of constraints are between 1 and 38, exceeding 10 in only 5 cases." In [18] the numerical tests are limited to: "The first case has 80 optimization variables [i.e., the size of the vector $\boldsymbol{x}$ ] and only bound constraints, while the second example is a generally constrained production optimization involving 20 optimization variables and 5 general constraints." Similar orders of magnitude for problem sizes appear in the numerical results presented in [1] and also in the book of Audet and Hare [2].

This indicates that (i) much of the literature on derivative-free minimization is concerned with unconstrained minimization or with bound-constraints on the variables, and (ii) many, if not all, proposed methods were designed (or, at least, demonstrated) only for small-scale problems. In contrast, the DFS method proposed here can handle any type of constraints for which a separate efficient constraints-compatibility-seeking algorithm is available and is capable of solving very large problems. In the matter of problem sizes, we discover here, admittedly with a very preliminary demonstration, that DFS can compete well with DFO on large problems. Since the constraintscompatibility-seeking algorithm forms part of the proposed DFS method, the method can use exterior initialization (that is initializing the iterations at any point in space). Furthermore, very large-scale problems can be accommodated.

The progressive barrier (PB) approach, described in Chapter 12 of the book [2], originally published in [1], is an alternative to the exterior penalty (EP) approach that we mention in Subsection 5.4 below. However, the PB differs from our DFS method, in spite of some similarities with it, as we explain in Subsection 5.5 below.

1.3 Earlier work on superiorization and the "guarantee problem"

A comprehensive overview of the state of the art and current research on superiorization appears in our continuously updated bibliography Internet page that currently contains 109 items [8]. Research works in this bibliography include a variety of reports ranging from new applications to new mathematical results on the foundations of superiorization. A special issue entitled: "Superiorization: Theory and Applications" of the journal Inverse Problems [11] contains several interesting papers on the theory and practice of SM, such as [6], [24], [29], [38] and [39], to name but a few. Later papers continue research on perturbation resilience, which lies at the heart of the SM, see, e.g., [3]. An early paper on bounded perturbation resilience is [4], a recent book containing results on the behavior of algorithms in the presence of summable perturbations is [42].

In $[12$, Section 3] we gave a precise definition of the "guarantee problem" of the SM. We wrote there:

The SM interlaces into a feasibility-seeking basic algorithm target function reduction steps. These steps cause the target function to reach lower values locally, prior to performing the next feasibility-seeking iterations. A mathematical 
guarantee has not been found to date that the overall process of the superiorized version of the basic algorithm will not only retain its feasibility-seeking nature but also preserve globally the target function reductions. We call this fundamental question of the SM "the guarantee problem of the SM" which is: "under which conditions one can guarantee that a superiorized version of a bounded perturbation resilient feasibility-seeking algorithm converges to a feasible point that has target function value smaller or equal to that of a point to which this algorithm would have converged if no perturbations were applied - everything else being equal."

Numerous works that are cited in [8] show that this global function reduction of the SM occurs in practice in many real-world applications. But until the guarantee problem of the SM is answered one wonders if the SM is just a successful heuristic or if there is a mathematical foundation for the accumulating reports on its performance success?" Except for a partial answer in [12] with the aid of the "concentration of measure" principle there are also the partial result of [13, Theorem 4.1] about strict Fejér monotonicity of sequences generated by an SM algorithm.

\subsection{Structure of the paper}

In Section 2 we present the basics of the superiorization methodology. We present our DFS algorithm in Section 3 and juxtapose it with an existing superiorization algorithm that uses derivative information. In Section 4 we present a tool (we call it a proximitytarget curve) for deciding which of two iterative methods is "better" for solving a particular problem. The experimental demonstration of our DFS algorithm appears in Section 5. In Section 6 we offer a brief discussion and some conclusions.

\section{The basics of the superiorization methodology}

We follow the approach of [28]. A word about terminology before we begin: The SM has been developed with the terminology presented here, see, e.g., [27]. Adhering to it will assist readers when referring to other publications and will contribute to separate it from similar notions that are used in optimization theory.

$\Omega$ denotes a nonempty set in the Euclidean space $\mathbb{R}^{J} . \mathbb{T}$ is a problem set; each problem $T \in \mathbb{T}$ is described by a particular set of constraints such as provided, for example, in (1). $\mathcal{P r}$ is a proximity function on $\mathbb{T}$ such that, for every $T \in \mathbb{T}, \mathcal{P} r_{T}$ : $\Omega \rightarrow \mathbb{R}_{+}$(nonnegative real numbers). $\mathcal{P} r_{T}(\boldsymbol{x})$ measures how incompatible $\boldsymbol{x}$ is with the constraints of $T$. A problem structure is a pair $(\mathbb{T}, \mathcal{P} r)$, where $\mathbb{T}$ is a problem set and $\mathcal{P} r$ is a proximity function on $\mathbb{T}$. For an $\boldsymbol{x} \in \Omega$, we say that $\boldsymbol{x}$ is $\varepsilon$-compatible with $T$ if $\mathcal{P} r_{T}(\boldsymbol{x}) \leq \varepsilon$. We assume that we have computer procedures that, for any $\boldsymbol{x} \in \mathbb{R}^{J}$, determine whether $\boldsymbol{x} \in \Omega$ and, for any $\boldsymbol{x} \in \Omega$ and $T \in \mathbb{T}$, calculate $\mathcal{P} r_{T}(\boldsymbol{x})$. In many applications, each problem $T \in \mathbb{T}$ is determined by a family of sets $\left\{C_{i}\right\}_{i=1}^{I}$, where each $C_{i}$ is a nonempty, often closed and convex, subset of $\Omega$ and the problem $T$ is to find a point that is in the intersection of the $C_{i}$.

We introduce $\Delta$, such that $\Omega \subseteq \Delta \subseteq \mathbb{R}^{J}$ and a target function $\phi: \Delta \rightarrow \mathbb{R}$, which is referred to as an optimization criterion in [28]. We assume that we have a computer procedure that, for any $\boldsymbol{x} \in \mathbb{R}^{J}$, determines whether $\boldsymbol{x} \in \Delta$ and, if so, calculates $\phi(\boldsymbol{x})$. 
An algorithm $\mathbf{P}$ for a problem structure $(\mathbb{T}, \mathcal{P} r)$ assigns to each problem $T \in \mathbb{T}$ a computable algorithmic operator $\mathbf{P}_{T}: \Delta \rightarrow \Omega$. For any initial point $\boldsymbol{x} \in \Omega, \mathbf{P}_{T}$ produces the infinite sequence $\left(\left(\mathbf{P}_{T}\right)^{k} \boldsymbol{x}\right)_{k=0}^{\infty}$ of points in $\Omega$. The next definition gives a name to the first element in a sequence $\left(\boldsymbol{x}^{k}\right)_{k=0}^{\infty}$ with $\mathcal{P} r_{T}\left(\boldsymbol{x}^{k}\right) \leq \varepsilon$.

Definition 1 The $\varepsilon$-output of a sequence

For a problem structure $(\mathbb{T}, \mathcal{P} r)$, a $T \in \mathbb{T}$, an $\varepsilon \in \mathbb{R}_{+}$and a sequence $R:=\left(\boldsymbol{x}^{k}\right)_{k=0}^{\infty}$ of points in $\Omega$, we use $O(T, \varepsilon, R)$ to denote the $\boldsymbol{x} \in \Omega$ that has the following properties: $\mathcal{P r}_{T}(\boldsymbol{x}) \leq \varepsilon$, and there is a nonnegative integer $K$ such that $\boldsymbol{x}^{K}=\boldsymbol{x}$ and, for all nonnegative integers $k<K, \mathcal{P} r_{T}\left(\boldsymbol{x}^{k}\right)>\varepsilon$. If there is such an $\boldsymbol{x}$, then it is unique. If there is no such $\boldsymbol{x}$, then we say that $O(T, \varepsilon, R)$ is undefined, otherwise it is defined.

If $R$ is an infinite sequence generated by a process that repeatedly applies $\mathbf{P}_{T}$, then $O(T, \varepsilon, R)$ is the output produced by that process when we add to it instructions that make it terminate as soon as it reaches a point that is $\varepsilon$-compatible with $T$. Roughly, we refer to $\mathbf{P}$ as a feasibility-seeking algorithm for a problem structure $(\mathbb{T}, \mathcal{P r})$ that arose from a particular application if, for all $T \in \mathbb{T}$ and $\varepsilon \in \mathbb{R}_{+}$of interest for the application, $O(T, \varepsilon, R)$ is defined for all infinite sequences $R$ generated by repeated applications $\mathbf{P}_{T}$. Each application of $\mathbf{P}_{T}$ is referred to as a feasibility-seeking step.

\section{Definition 2 Strong perturbation resilience}

An algorithm $\mathbf{P}$ for a problem structure $(\mathbb{T}, \mathcal{P} r)$ is said to be strongly perturbation resilient if, for all $T \in \mathbb{T}$,

1. there is an $\varepsilon \in \mathbb{R}_{+}$such that $O\left(T, \varepsilon,\left(\left(\mathbf{P}_{T}\right)^{k} \boldsymbol{x}\right)_{k=0}^{\infty}\right)$ is defined for every $\boldsymbol{x} \in \Omega$;

2. for all $\varepsilon \in \mathbb{R}_{+}$such that $O\left(T, \varepsilon,\left(\left(\mathbf{P}_{T}\right)^{k} \boldsymbol{x}\right)_{k=0}^{\infty}\right)$ is defined for every $\boldsymbol{x} \in \Omega$, we also have that $O\left(T, \varepsilon^{\prime}, R\right)$ is defined for every $\varepsilon^{\prime}>\varepsilon$ and for every sequence $R=\left(\boldsymbol{x}^{k}\right)_{k=0}^{\infty}$ of points in $\Omega$ generated by

$$
\boldsymbol{x}^{k+1}=\mathbf{P}_{T}\left(\boldsymbol{x}^{k}+\beta_{k} \boldsymbol{v}^{k}\right), \text { for all } k \geq 0,
$$

where $\beta_{k} \boldsymbol{v}^{k}$ are bounded perturbations, meaning that the sequence $\left(\beta_{k}\right)_{k=0}^{\infty}$ of nonnegative real numbers is summable (that is, $\sum_{k=0}^{\infty} \beta_{k}<\infty$ ), the sequence $\left(\boldsymbol{v}^{k}\right)_{k=0}^{\infty}$ of vectors in $\mathbb{R}^{J}$ is bounded and, for all $k \geq 0, \boldsymbol{x}^{k}+\beta_{k} \boldsymbol{v}^{k} \in \Delta$.

Sufficient conditions for strong perturbation resilience appeared in [28, Theorem 1]. Here and elsewhere, while the $\beta_{k}$ s are nonnegative real numbers, one should disallow the trivial case in which all $\beta_{k}$ s are zero because obviously that would completely nullify all perturbations. Notice that for most feasibility-seeking algorithms we have $\lim _{k \rightarrow \infty} \mathcal{P r}\left(\left(\mathbf{P}_{T}\right)^{k} \boldsymbol{x}\right)=\inf _{\boldsymbol{y}} \mathcal{P} r(\boldsymbol{y})$, which means that superiorization of a strongly perturbation resilient feasibility-seeking algorithm will generate an infinite perturbed sequence such that $\lim _{k \rightarrow \infty} \mathcal{P r}\left(\boldsymbol{x}^{k}\right)=\inf _{\boldsymbol{x}} \operatorname{Pr}(\boldsymbol{x})$.

With respect to the target function $\phi: \Delta \rightarrow \mathbb{R}$, we adopt the convention that a point in $\Delta$ for which the value of $\phi$ is smaller is considered superior to a point in $\Delta$ for which the value of $\phi$ is larger. The essential idea of the SM is to make use of the perturbations of (3) to transform a strongly perturbation resilient algorithm 
that seeks a constraints-compatible solution (referred to as the Basic Algorithm) into a superiorized version whose outputs are equally good from the point of view of constraints-compatibility, but are superior (not necessarily optimal) with respect to the target function $\phi$. This can be done by making use of the following concept.

\section{Definition 3 [28] Nonascending vector}

Given a function $\phi: \Delta \rightarrow \mathbb{R}$ and a point $\boldsymbol{y} \in \mathbb{R}^{J}$, we say that a $\boldsymbol{d} \in \mathbb{R}^{J}$ is a nonascending vector for $\phi$ at $\boldsymbol{y}$ if $\|\boldsymbol{d}\| \leq 1$ and there is a $\delta>0$ such that

$$
\text { for all } \lambda \in[0, \delta] \text { we have } \phi(\boldsymbol{y}+\lambda \boldsymbol{d}) \leq \phi(\boldsymbol{y}) \text {. }
$$

Obviously, the zero vector $\mathbf{0}$ (all components are 0) is always such a vector, but for the SM to work we need a strict inequality to occur in (4) frequently enough. Generation of nonascending vectors, used for target function reduction steps, has been based mostly on the following theorem or its variants such as [21, Theorem 1] and [22, unnumbered Theorem on page 7], which provide sufficient conditions for a nonascending vector.

Theorem 1 [28, Theorem 2]. Let $\phi: \mathbb{R}^{J} \rightarrow \mathbb{R}$ be a convex function and let $\boldsymbol{x} \in$ $\mathbb{R}^{J}$. Let $\boldsymbol{g} \in \mathbb{R}^{J}$ satisfy the property: For $1 \leq j \leq J$, if the $j$ th component $g_{j}$ of $\boldsymbol{g}$ is not zero, then the partial derivative $\frac{\partial \phi}{\partial x_{j}}(\overline{\boldsymbol{x}})$ of $\phi$ at $\boldsymbol{x}$ exists and its value is $g_{j}$. Define $\boldsymbol{d}$ to be the zero vector if $\|\boldsymbol{g}\|=0$ and to be $-\boldsymbol{g} /\|\boldsymbol{g}\|$ otherwise. Then $\boldsymbol{d}$ is a nonascending vector for $\phi$ at $\boldsymbol{x}$.

In order to use this theorem, $\phi$ must have at least one calculable partial derivative (which is nonzero) at points in the domain of $\phi$. Otherwise, the theorem would apply only to the zero vector, which is a useless nonascending vector because it renders the $\mathrm{SM}$ ineffective. If $\phi$ is not differentiable at some points we can just take $\boldsymbol{v}^{k}=0$ at those points, but $\phi$ needs to be differentiable at "enough" points for the superiorization to be effective. To enable application of the SM to target functions that have no calculable partial derivatives or subgradients, we proposed in [10] to search for a point in the neighborhood of $\boldsymbol{x}$ at which the target function exhibits nonascent by comparing function values at points of a fixed distance from $\boldsymbol{x}$ along the space coordinates. To obtain a sequence of nonascending points without making use of Theorem 1, we replaced in [10] the notion of a nonascending vector by the following alternative notion.

\section{Definition 4 [10] Nonascending $\delta$-bound direction}

Given a target function $\phi: \Delta \rightarrow \mathbb{R}$ where $\Delta \subseteq \mathbb{R}^{J}$, a point $\boldsymbol{y} \in \Delta$, and a positive $\delta \in \mathbb{R}$, we say that $\boldsymbol{d} \in \mathbb{R}^{J}$ is a nonascending $\delta$-bound direction for $\phi$ at $\boldsymbol{y}$ if $\|\boldsymbol{d}\| \leq \delta, \boldsymbol{y}+\boldsymbol{d} \in \Delta$ and $\phi(\boldsymbol{y}+\boldsymbol{d}) \leq \phi(\boldsymbol{y})$. The collection of all such vectors is called a nonascending $\delta$-ball and is denoted by $\mathcal{B}_{\delta, \phi}(\boldsymbol{y})$, that is,

$$
\mathcal{B}_{\delta, \phi}(\boldsymbol{y}):=\left\{\boldsymbol{d} \in \mathbb{R}^{J} \mid\|\boldsymbol{d}\| \leq \delta,(\boldsymbol{y}+\boldsymbol{d}) \in \Delta, \phi(\boldsymbol{y}+\boldsymbol{d}) \leq \phi(\boldsymbol{y})\right\}
$$

The zero vector is contained in each nonascending $\delta$-ball, that is, $\mathbf{0} \in \mathcal{B}_{\delta, \phi}(\boldsymbol{y})$ for each $\delta>0$ and $\boldsymbol{y} \in \Delta$. The purpose of this definition is to allow the use, as a direction of target function decrease, of any vector $\boldsymbol{d} \in \mathbb{R}^{J}$ for which $\phi(\boldsymbol{y}+\boldsymbol{d}) \leq \phi(\boldsymbol{y})$ holds locally only for $\boldsymbol{d}$, and not throughout a certain interval as in Definition 3. The vector $\boldsymbol{d}$ depends on the value of $\delta$ and they may be determined simultaneously in the superiorization process, as seen below. This kind of nonascent was referred to as 
local nonascent in [10, Subsection 2.3]. Obviously, local nonascent is a more general notion since every nonascending vector according to Definition 3 is also a nonascending $\delta$-bound direction according to Definition 4 but not vice versa. The advantage of this notion is that it is detectable by using only function value calculations.

The following easily-proved proposition unifies these approaches in the convex case.

Proposition 1 Let $\phi: \mathbb{R}^{J} \rightarrow \mathbb{R}$ be a convex function and let $\boldsymbol{x} \in \mathbb{R}^{J}$. If $\boldsymbol{d} \in \mathbb{R}^{J}$ is a nonascending $\delta$-bound direction for $\phi$ at $\boldsymbol{x}$, then either $\boldsymbol{d}=\mathbf{0}$ (and hence $\boldsymbol{d}$ is a nonascending vector for $\phi$ at $\boldsymbol{x}$ ) or $\boldsymbol{d} /\|\boldsymbol{d}\|$ is a nonascending vector for $\phi$ at $\boldsymbol{x}$.

The idea of calculating $\delta$ (equivalently, the step-size $\gamma_{\ell}$ in the superiorized algorithms presented in the next section) simultaneously with a direction of nonascent appeared in a completely different way in [33], where they use an additional internal loop of a penalized minimization to calculate the direction of nonascent; see also [34].

\section{Specific superiorization approaches}

This section presents two specific approaches to superiorizing a Basic Algorithm that operates by repeated applications of an algorithmic operator $\mathbf{P}_{T}$ starting from some initial point. The first approach produces the superiorized version that is named Algorithm 1 below, it has been published in the literature previously [28, page 5537]. The second approach, named Algorithm 2 below, is novel to this paper.

The two superiorized versions have some things in common. They are both iterative procedures in which $k$ is used as the iteration index. The first two steps of both algorithms sets $k$ to 0 and $\boldsymbol{x}^{0}$ to a given initial vector $\overline{\boldsymbol{x}} \in \Delta$. They both assume that we have available a summable sequence $\left(\gamma_{\ell}\right)_{\ell=0}^{\infty}$ of nonnegative real numbers, not all of them zero, (for example, $\gamma_{\ell}=a^{\ell}$, where $0<a<1$ ). In Step 3 of both algorithms, $\ell$ is initialized to -1 (this is acceptable since $\ell$ is increased by 1 before the first time $\gamma_{\ell}$ is used). In both algorithms the iterative step that produces $\boldsymbol{x}^{k+1}$ from $\boldsymbol{x}^{k}$, as in (3), is specified within a repeat loop that first performs a user-specified number, $N$, of perturbation steps followed by one feasibility-seeking step that uses the algorithmic operator $\mathbf{P}_{T}$. In more detail, the repeat loop in each of the algorithms has the following form. After initializing the loop index $n$ to 0 and setting $\boldsymbol{x}^{k, 0}$ to $\boldsymbol{x}^{k}$, it produces one-by-one $\boldsymbol{x}^{k, 1}, \boldsymbol{x}^{k, 2}, \ldots, \boldsymbol{x}^{k, N}$ (these are the iterations of the perturbation steps), followed by producing $\boldsymbol{x}^{k+1}=\mathbf{P}_{T} \boldsymbol{x}^{k, N}$ (the feasibility-seeking step). The difference between the two algorithms is in how they perform the perturbations for getting from $\boldsymbol{x}^{k, n}$ to $\boldsymbol{x}^{k, n+1}$.

We state an important property of Algorithm 1; for a proof see [28, Section II.E].

Theorem 2 Suppose that the algorithm $\mathbf{P}$ for a problem structure $(\mathbb{T}, \mathcal{P} r)$ is strongly perturbation resilient. Suppose further that $T \in \mathbb{T}$ and $\varepsilon \in \mathbb{R}_{+}$are such that $O\left(T, \varepsilon,\left(\left(\mathbf{P}_{T}\right)^{k} \boldsymbol{x}\right)_{k=0}^{\infty}\right)$ is defined for every $\boldsymbol{x} \in \Omega$. It is then the case that $O\left(T, \varepsilon^{\prime}, R\right)$ is defined for every $\varepsilon^{\prime}>\varepsilon$ and every sequence $R=\left(\boldsymbol{x}^{k}\right)_{k=0}^{\infty}$ produced by Algorithm 1.

The pseudo-code of Algorithm 1 does not specify how the nonascending vector in Step 8 is to be selected. In publications using Algorithm 1, such details are usually based on a variant of Theorem (1), resulting in a not derivative-free algorithm. 


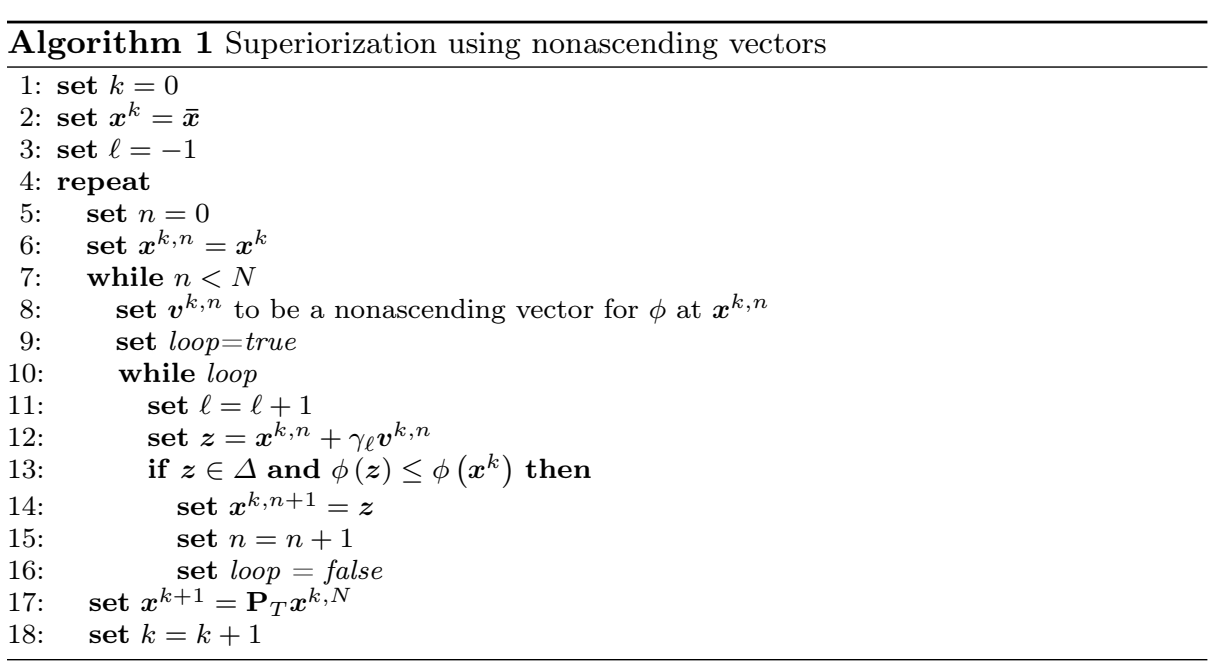

For the specification of Algorithm 2 we let, for $1 \leq j \leq J$, $\mathbf{e}^{j}$ be the vector in $\mathbb{R}^{J}$ all of whose components are 0 , except for the $j$ th component, which is 1 . The set of coordinate directions is defined as $\Gamma:=\left\{\mathbf{e}^{j} \mid 1 \leq j \leq J\right\} \cup\left\{-\mathbf{e}^{j} \mid 1 \leq j \leq J\right\}$. We assume that $\left(\mathbf{c}^{m}\right)_{m=0}^{\infty}$ is a given sequence of coordinate directions such that any subsequence of length $2 \bar{J}$ contains $\Gamma$.

The component-wise approach indicated in Algorithm 2 has been presented previously in the literature as a stand-alone optimization method, referred to as "compass search"; see the review paper by Kolda et al. [31], which is an excellent source for direct search methods that do not explicitly use derivatives. Here we apply it to superiorize a feasibility-seeking algorithm, rather than as a stand-alone optimization method.

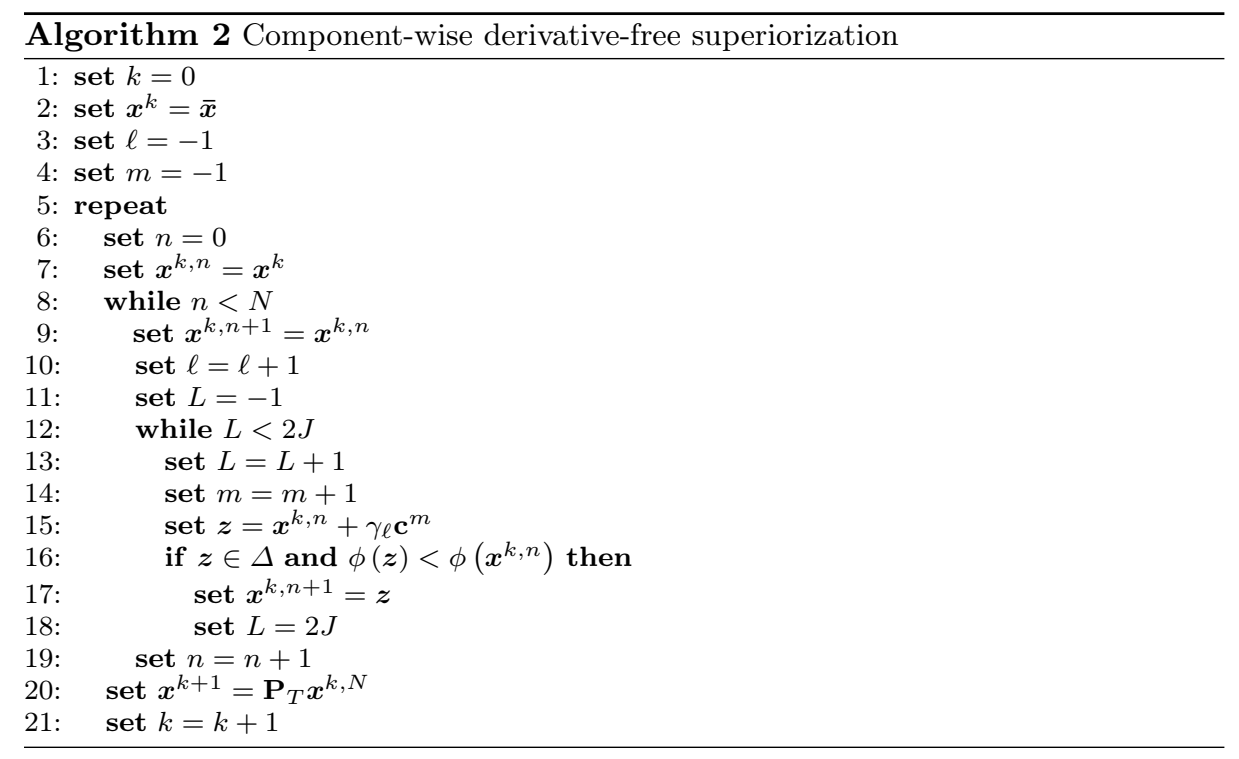


We make the following comments:

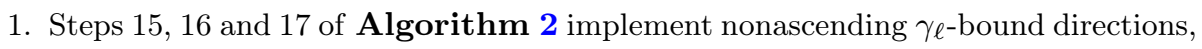
as in Definition 4. In doing so, Algorithm 2 realizes in a component-wise manner the algorithmic framework of [10] (specifically, as expressed in Steps 7 and 8 of Algorithm 1 in that paper).

2. No partial derivatives are used by Algorithm 2.

3. Step 16 of Algorithm 2 is similar to Step 13 of Algorithm 1. One difference is the use of strict inequality in Algorithm 2, the reason for this is that it was found advantageous in some applications of the algorithm. In addition, the while loop due to Step 12 of Algorithm 2 is executed at most $2 J$ times, but there is no upper bound on the (known to be finite) number of executions of the while loop due to Step 10 of Algorithm 1. Also, it follows from the pseudo-code of Algorithm 2 that, for all $k \geq 0$ and $0 \leq n \leq N, \phi\left(\boldsymbol{x}^{k, n}\right)<\phi\left(\boldsymbol{x}^{k}\right)$, even though there is no explicit check for this as in Step 13 of Algorithm 1.

4. Algorithm 2 has the essential property that it cannot get stuck in a particular iteration $k$ because the value of $L$ increases in an execution of the while loop of Step 13 and the value of $n$ increases in an execution of the while loop of Step 8.

5. Algorithm 2 shares with Algorithm 1 the important property in Theorem 2. Stated less formally: "For a strongly perturbation resilient algorithm, if for all initial points from $\Omega$ the infinite sequence produced by an algorithm contains an $\varepsilon$-compatible point, then all perturbed sequences produced by the superiorized version of the algorithm contain an $\varepsilon^{\prime}$-compatible point, for any $\varepsilon^{\prime}>\varepsilon$."

6. At present there is no mathematical proof to guarantee that the output of a superiorized version of a constraints-compatibility-seeking algorithm will have smaller target function $\phi$ value than the output by the same constraints-compatibilityseeking algorithm without perturbations, everything else being equal. A partial mathematical result toward coping with this lacuna, in the framework of weak superiorization, is provided by Theorem 4.1 in [13]. ${ }^{1}$

\section{The proximity-target curve}

We now give a tool for deciding which of two iterative methods is "better" for solving a particular problem. Since an iterative method produces a sequence of points, our definition is based on such sequences. Furthermore, since we are interested in the values of two functions (proximity function and target function) at each of the points, the efficacy of the behavior of the iterative method can be represented by a curve in two-dimensional space, defined as the proximity target curve below. It indicates the target value for any achieved proximity value. This leads to the intuitive concept of an algorithm being "better" than another one, if its proximity target curve is below that of the other one (that is, the target value for it is always smaller than the target value of the other one for the same proximity value). Such may not always be the case, the two proximity curves may cross each other, providing us with intervals of proximity values within which one or the other method is better.

This way of thinking is commonly used in many fields of science in situations where it is desirable to obtain an object for which the values of two evaluating functions are

1 The approach followed in the present paper was termed strong superiorization in [13, Section 6] and [7] to distinguish it from weak superiorization, wherein asymptotic convergence to a point in $C$ is studied instead of $\varepsilon$-compatibility. 
simultaneously small. A prime example is in estimation theory where we desire an estimation method with both small bias and small variance. More specifically, and nearer to the application areas of the authors, is the concept of a receiver operating characteristics (ROC) curve that illustrates the diagnostic ability of a binary classifier system as its discrimination threshold is varied. It is created by plotting the true positive rate against the false positive rate at various threshold settings. One classifier system is considered "better" than the other one if its ROC curve is above that of the other one; but, just as for our proximity-target curves, the ROC curves for two classifier systems may cross each other. There are many publications on the role of ROC curves in the evaluation of medical imaging techniques; see, for example, [35,41]. Their use for image reconstruction algorithm evaluation is discussed, for example, in [15].

For incarnations of the definitions given in this section, the reader may wish to look ahead to Figure 2. That figure illustrates the notions discussed in this section for two particular finite sequences $R:=\left(\boldsymbol{x}^{k}\right)_{k=K_{l o}}^{K_{h i}}$ and $S:=\left(\boldsymbol{y}^{k}\right)_{k=L_{l o}}^{L_{h i}}$. The details of how those sequences were produced are given below in Subsection 5.6.

\section{Definition 5 Monotone proximity of a finite sequence}

For a problem structure $(\mathbb{T}, \mathcal{P} r)$, a $T \in \mathbb{T}$, positive integers $K_{l o}$ and $K_{h i}>K_{l o}$, the finite sequence $R:=\left(\boldsymbol{x}^{k}\right)_{k=K_{l_{0}}}^{K_{h i}}$ of points in $\Omega$ is said to be of monotone proximity if for $K_{l o}<k \leq K_{h i}, \mathcal{P} r_{T}\left(x^{k-1}\right)>\mathcal{P} r_{T}\left(x^{k}\right)$.

\section{Definition 6 The proximity-target curve of a finite sequence}

For a problem structure $(\mathbb{T}, \mathcal{P} r)$, a $T \in \mathbb{T}$, a target function $\phi: \Omega \rightarrow \mathbb{R}$, positive integers $K_{l o}$ and $K_{h i}>K_{l o}$, let $R:=\left(\boldsymbol{x}^{k}\right)_{k=K_{l o}}^{K_{h i}}$ be a sequence of monotone proximity. Then the proximity-target curve $P \subseteq \mathbb{R}^{2}$ associated with $R$ is uniquely defined by:

1. For $K_{l o} \leq k \leq K_{h i},\left(\mathcal{P} r_{T}\left(\boldsymbol{x}^{k}\right), \phi\left(\boldsymbol{x}^{k}\right)\right) \in P$.

2. The intersection $\left\{(y, x) \in \mathbb{R}^{2}: \mathcal{P} r_{T}\left(\boldsymbol{x}^{k}\right) \leq y \leq \mathcal{P} r_{T}\left(\boldsymbol{x}^{k-1}\right)\right\} \cap P$ is the line segment from $\left(\mathcal{P} r_{T}\left(\boldsymbol{x}^{k-1}\right), \phi\left(\boldsymbol{x}^{k-1}\right)\right)$ to $\left(\mathcal{P} r_{T}\left(\boldsymbol{x}^{k}\right), \phi\left(\boldsymbol{x}^{k}\right)\right)$.

\section{Definition 7 Comparison of proximity-target curves of sequences}

For a problem structure $(\mathbb{T}, \mathcal{P} r)$, a $T \in \mathbb{T}$, a target function $\phi: \Omega \rightarrow \mathbb{R}$, positive integers $K_{l o}, K_{h i}>K_{l o}, L_{l o}, L_{h i}>L_{l o}$, let $R:=\left(\boldsymbol{x}^{k}\right)_{k=K_{l o}}^{K_{h i}}$ and $S:=\left(\boldsymbol{y}^{k}\right)_{k=L_{l o}}^{L_{h i}}$ be sequences of points in $\Omega$ of monotone proximity for which $P$ and $Q$ are their respective associated proximity-target curves. Define

$$
\begin{aligned}
t & :=\max \left(\mathcal{P} r_{T}\left(\boldsymbol{x}^{K_{h i}}\right), \mathcal{P} r_{T}\left(\boldsymbol{y}^{L_{h i}}\right)\right), \\
u & :=\min \left(\mathcal{P} r_{T}\left(\boldsymbol{x}^{K_{l o}}\right), \mathcal{P} r_{T}\left(\boldsymbol{y}^{L_{l o}}\right)\right) .
\end{aligned}
$$

Then $R$ is better targeted than $S$ if:

1. $t \leq u$ and

2. for any real number $h$, if $t \leq h \leq u,(h, v) \in P$ and $(h, w) \in Q$, then $v \leq w$.

Let us see how this last definition translates into something that is intuitively desirable. Suppose that we have an iterative algorithm that produces a sequence, $\boldsymbol{y}^{0}, \boldsymbol{y}^{1}, \boldsymbol{y}^{2}, \cdots$, of which $S:=\left(\boldsymbol{y}^{k}\right)_{k=L_{l o}}^{L_{h i}}$ is a subsequence. An alternative algorithm that produces a sequence of points of which $R:=\left(\boldsymbol{x}^{k}\right)_{k=K_{l o}}^{K_{h i}}$ is a subsequence that is 
better targeted than $S$ has a desirable property: Within the range $[t, u]$ of proximity values, the point that is produced by the alternative algorithm with that proximity value, is likely to have lower (and definitely not higher) value of the target function as the point with that proximity value that is produced by the original algorithm. This property is stronger than what we stated before, namely that superiorization produces an output that is equally good from the point of view of proximity, but is superior with respect to the target function. Here the single output determined by a fixed $\varepsilon$ is replaced by a set of potential outputs for any $\varepsilon \in[t, u]$.

\section{Experimental demonstration of derivative-free component-wise superiorization}

\subsection{Goal and general methodology}

Our goal is to demonstrate that component-wise superiorization (Algorithm 2) is a viable efficient DFS method to handle data of constrained-minimization problems (that is, a target function and a set of constraints), when the target function has no calculable partial derivatives.

To ensure the meaningfulness and worthiness of our experiments, we generate the constraints and choose a target function, that has no calculable partial derivatives, inspired by an application area of constrained optimization, namely image reconstruction from projections in computerized tomography (CT).

For the so-obtained data we consider two runs of Algorithm 2, one with and the other without the component-wise perturbation steps. To be exact, by "without perturbation" we mean that Steps $10-18$ in Algorithm 2 are deleted so that $\boldsymbol{x}^{k, N}=$ $\boldsymbol{x}^{k}$, which amounts to running the feasibility-seeking basic algorithm $\mathbf{P}_{T}$ without any perturbations. Everything else is equal in the two runs, such as the initialization point $\overline{\boldsymbol{x}}$ and all parameters associated with the application of the feasibility-seeking basic algorithm in Step 20. The results are presented below by plots of proximity-target curves that show that the target function values of Algorithm 2 when run "with perturbations" are systematically lower than those of the same algorithm without the component-wise perturbations.

The numerical behavior of Algorithm 2, as demonstrated by our experiment, makes it a meritorious choice for superiorization in situations involving a derivativefree target function and a set of constraints.

To reach the goal described above we proceed in the following stages.

1. Specification of a problem structure $(\mathbb{T}, \mathcal{P} r)$ for the experimental demonstration, and generation of constraints, simulated from the application of image reconstruction from projections in computerized tomography.

2. Choice of a $\Delta$ and a derivative-free target function $\phi$ for the experiment.

3. Specification of the algorithmic operator $\mathbf{P}_{T}$ to be used in Algorithm 2. This is chosen so that the Basic Algorithm that operates by repeated applications of $\mathbf{P}_{T}$ is a standard sequential iterative projections method for feasibility-seeking of systems of linear equations; a version of the Algebraic Reconstruction Techniques (ART) [25, Chapter 11] that is equivalent to Kaczmarz's projections method [30].

4. Specification of algorithmic details and parameters, such as $N$ and $\gamma_{\ell}$ in Algorithm 2. 
5.2 Problem selection, constraints generation and choices of $\Delta$ and of the target function

We generate the constraints and chose a target function from the application area of image reconstruction from projections in computerized tomography $(\mathrm{CT}) .^{2}$ The problem structure $(\mathbb{T}, \mathcal{P r})$ for our demonstration has been used in the literature for comparative evaluations of various algorithms for CT $[22,25,28,36]$. It is of the type described in Section 1 by (1) and (2). Specifically, vectors $\boldsymbol{x}$ in $\Omega=\mathbb{R}^{J}$ represent twodimensional (2D) images, with each component of $\boldsymbol{x}$ representing the density assigned to one of the pixels in the image. We use $J=235,225$, thus each $\boldsymbol{x}$ represents a $485 \times 485$ image. Our test image (phantom) is represented by the vector $\hat{\boldsymbol{x}}$, that is a digitization of a picture of a cross-section of a human head. The picture underlying the digitization is geometrically defined so that it has a value at every point of the planar cross-section; see [25, Sections 4.1-4.4 and 5.2].

In the problem $T$ that we use for our illustration, each index $i=1,2, \ldots, I$ is associated with a line across the image and the corresponding $\boldsymbol{d}^{i}$ is a vector in $\mathbb{R}^{J}$, whose $j$ th component is the length of intersection of that line with the $j$ th of the $J$ pixels. There are $I=498,960$ such lines (organized into 720 divergent projections with 693 lines in each; similar to the standard geometry in [25] but with more lines in each projection). The $h_{i}$ have been calculated by simulating the behavior of CT scanning of the head cross-section [25, Section 4.5]. In particular, the projection data were simulated using the underlying picture (rather than its digitization) and incorporate the stochastic nature (noisiness) of data collection in CT (based on 1,000,000 photons for estimating every line integral). All the above was generated using the SNARK14 programming system for the reconstruction of 2D images from 1D projections [16], giving rise to a system of linear equations (1). For the resulting $T$, we calculated that the proximity of the phantom to the generated constraints is $\mathcal{P} r_{T}(\hat{\boldsymbol{x}})=6.4192$, which is not zero due to the phantom being a digitization of the underlying picture and the noise incorporated into the calculation the line integrals $h_{i}$.

For our demonstration we make the simplest choice for $\Delta$, namely, $\Delta=\Omega=$ $\mathbb{R}^{J}$. Our choice of the target function $\phi$ is as follows. We index the pixels (i.e., the components of a vector $\boldsymbol{x}$ ) by $j$ and let $\Theta$ denote the set of all indices of pixels that are not in the rightmost column or the bottom row of the $2 \mathrm{D}$ pixel array that displays that vector as an image. For any pixel with index $j \in \Theta$, let $r(j)$ and $b(j)$ be the index of the pixel to its right and below it in the $2 \mathrm{D}$ pixel array, respectively. Denoting by med the function that selects the median value of its three arguments, we define

$$
\phi(\boldsymbol{x}):=\sum_{j \in \Theta} \sqrt{\left|x_{j}-\operatorname{med}\left\{x_{j}, x_{r(j)}, x_{b(j)}\right\}\right|} .
$$

This function can be considered as an alternative to total variation for measuring the roughness in an image represented by the vector $\boldsymbol{x}$. It has been selected for the experiments demonstrating the behavior of derivative-free superiorization because finding partial derivatives for it is problematic. On the other hand, when only one pixel value (that is, only one component of the vector) is changed in vector $\boldsymbol{x}$ to get another vector $\boldsymbol{y}$, then it is possible to obtain $\phi(\boldsymbol{y})$ from $\phi(\boldsymbol{x})$ by computing only three of the terms

\footnotetext{
2 The term projection has in this field a different meaning than in convex analysis. It stands for a set of estimated line integrals through the image that has to be reconstructed, see [25, page 3].
} 
in the summation on the right-hand side of (7). These observations indicate that the use of the derivative-free approach of Steps 10-18 in Algorithm 2 is a viable option whereas Step 8 of Algorithm 1 is hard to perform unless the trivial nonascending vector $\boldsymbol{v}^{k, n}=\mathbf{0}$ is selected, which is ineffective. For our chosen phantom we calculated $\phi(\hat{\boldsymbol{x}})=2,048.57$.

\subsection{The algorithmic operator $\mathbf{P}_{T}$}

Our chosen operator, mapping $\boldsymbol{x}$ into $\mathbf{P}_{T} \boldsymbol{x}$, is specified by Algorithm 3. It depends on a real parameter $\lambda \in(0,2)$ in its Step 4 .
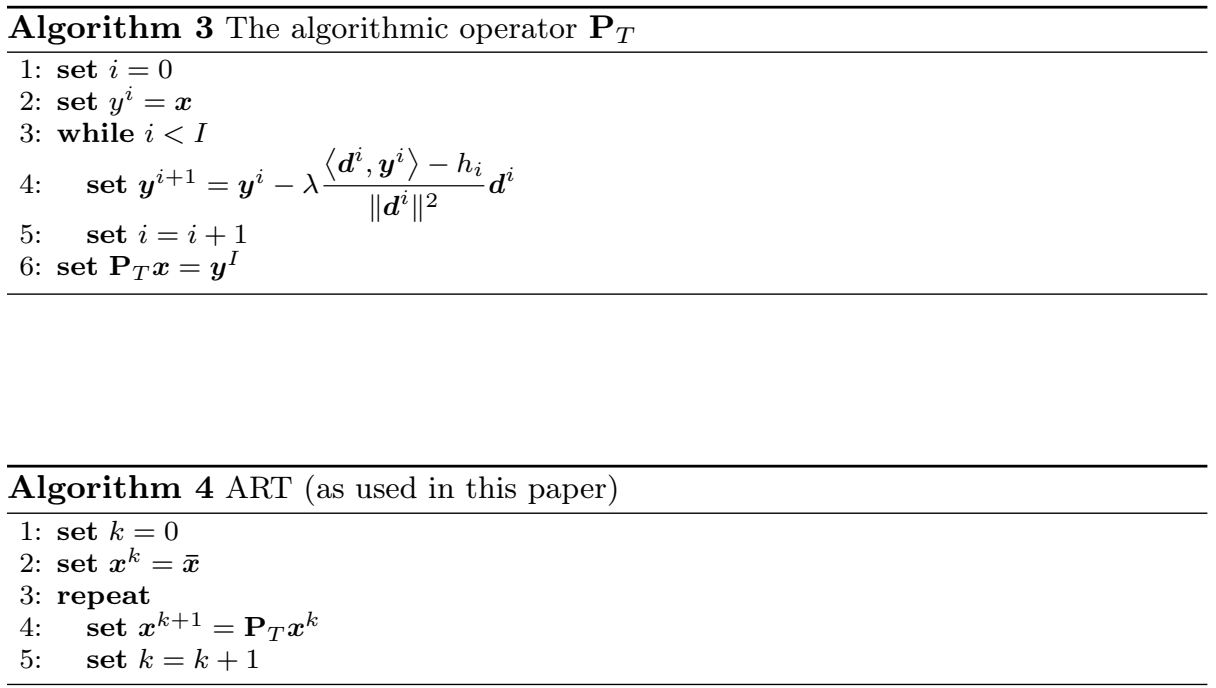

Algorithm 4 is a special case of the general class of Algebraic Reconstruction Techniques as discussed in [25, Chapter 11] and is, for $\lambda=1$, equivalent to the original method of Kaczmarz in the seminal paper [30]. For further references on Kaczmarz's method and the Algebraic Reconstruction Techniques see, e.g., [5, page 220], [9, Section 2 ] and [26]. Note that Algorithm 4 (ART) can be obtained from either Algorithm 1 or Algorithm 2 by removing the perturbation steps in their while loops.

5.4 A comment about the exterior penalty function approach to derivative-free constrained minimization

One possibility for doing a derivative-free constrained minimization algorithm is to follow the option of using the exterior penalty (EP) function approach mentioned in [14, Chapter 13, Section 13.1, page 242] as applied to the constrained problem

$$
\min \left\{\phi(\boldsymbol{x}) \mid\left\langle\boldsymbol{d}^{i}, \boldsymbol{x}\right\rangle=h_{i}, \quad i=1,2, \ldots, I\right\}
$$


where $\phi$ is as in (7) and the constraints are as in (1). With a user-selected penalization parameter $\eta$, the exterior penalty function approach replaces the constrained minimization problem (8) by the penalized unconstrained minimization:

$$
\begin{gathered}
\min \left\{\psi(\boldsymbol{x}) \mid \boldsymbol{x} \in \mathbb{R}^{J}\right\}, \\
\psi(\boldsymbol{x}):=\phi(\boldsymbol{x})+\eta \mathcal{P} r_{T}(\boldsymbol{x}),
\end{gathered}
$$

with $\phi$ as in (7) and $\mathcal{P r}_{T}(\boldsymbol{x})$ as defined in (2). By applying the coordinate-search method of [14, Algorithm 7.1] to the penalized unconstrained minimization problem (9)-(10), we get the next algorithm.

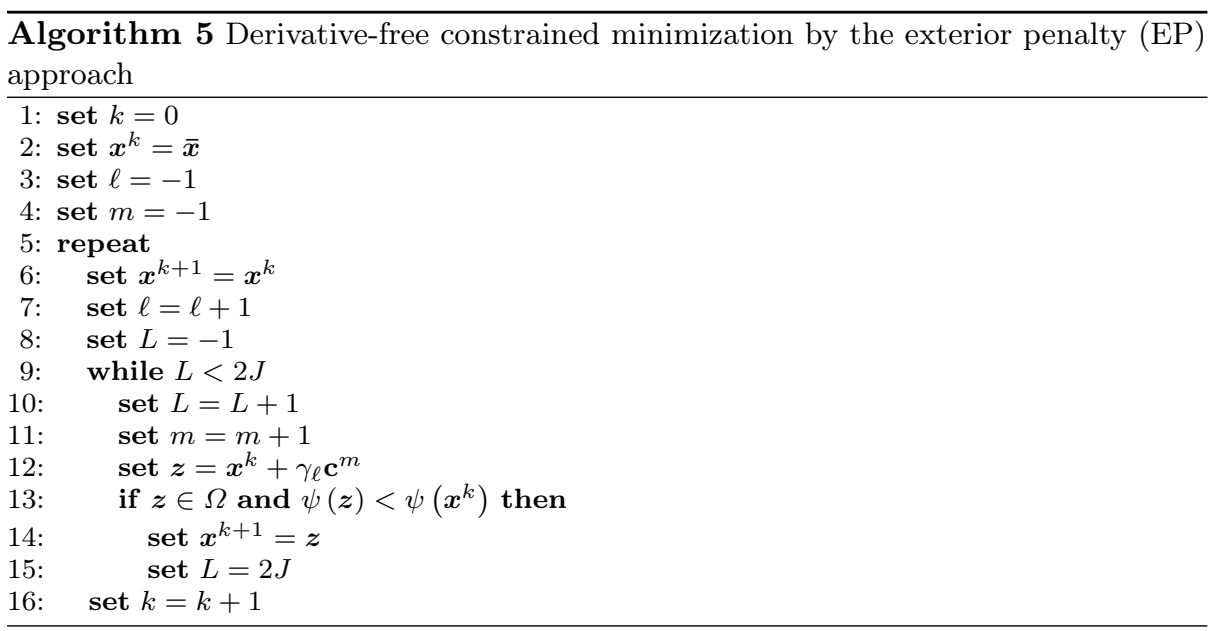

From the point of view of keeping the computational cost low, Algorithm 5 can be much more of a challenge than Algorithm 2. The reason for this has been indicated when we have stated, near the end of Subsection 5.2, that if only one component is changed in vector $\boldsymbol{x}$ to get another vector $\boldsymbol{y}$, then it is possible to obtain $\phi(\boldsymbol{y})$ from $\phi(\boldsymbol{x})$ by computing only three of the terms in the summation on the right-hand side of (7). When we use $\psi$ in (10) instead of $\phi$, there seems to be a need for many more computational steps. This is because the number of terms that change on the righthand side of (2) due to a change in one component of $\boldsymbol{x}$ is of the order of 1,000 for the dataset described in Subsection 5.2 (in the language of image reconstruction from projections, there is at least one line $i$ in each of of the 720 projections for which there is a change in value of $\left\langle\boldsymbol{d}^{i}, \boldsymbol{x}\right\rangle$ due to changing one component of $\boldsymbol{x}$ ). Thus our advocated approach of component-wise superiorization in Algorithm 2 is likely to be orders of magnitude faster for our application area than the more traditional approach of derivative-free constrained minimization by the exterior penalty (EP) approach in Algorithm 5.

5.5 The progressive barrier (PB) approach

The progressive barrier (PB) approach, described in Chapter 12 of [2], was originally published in [1] wherein the history of the approach as a development of the earlier filter 
methods of Fletcher and Leyffer [20] is succinctly described. It is an alternative to the exterior penalty (EP) approach, mentioned above, therefore, we briefly describe it here and point out how it differs from our DFS. The PB approach bears some similarities to our DFS but differs from it in a way that explains why the DFS will be advantageous for large-scale problems.

No penalty is used in the PB approach. Instead of combining the constraints with the target function it uses a particular "constraint violation function" $h(x)$ [2, Definition 12.1] alongside with the target function $\phi(x)$ so that the iterates appear in an $h$ versus $\phi$ plot called "a filter", based on the pairs of their $h$ and $\phi$ values. The constraint violation function of $\mathrm{PB}$ is similar in nature to our "proximity function" and the $h$ versus $\phi$ filter plot of $\mathrm{PB}$ is similar to our proximity-target curve, both mentioned above. The difference between the PB approach of [1] and our DFS lies in how these objects are used. The PB optimization algorithm defines at each iteration what is a "success" or a "failure" of an iterate based on the current filter plot, and decides accordingly what will be the next iterate. We do not bring the full details here but, in a nutshell, the PB optimization algorithm performs at each iteration sophisticated searches of both the target function values and the constraint violation function values.

In contrast with the PB approach, the DFS, investigated here, uses the world-view of superiorization. It uses a feasibility-seeking algorithm whose properties are already known and perturbs its iterates without losing its feasibility-seeking ability and properties. The component-wise derivative free search for a locally nonascending direction of the target function is done in a manner that makes it a perturbation to which the feasibility-seeking algorithm is resilient, i.e., that allows the underlying feasibilityseeking algorithm retain its feasibility-seeking nature. Thus, the DFS method searches only the target function in a derivative-free fashion and does automatic feasibilityseeking steps that actively reduce the proximity function. In this work the constraints are linear and the feasibility-seeking algorithm proceeds by performing orthogonal projections onto the hyperplanes that constitute the constraints sets). This means, on the face of it, an advantage for the DFS in handling large-scale problems because no expensive additional time and computing resources are needed for the feasibility-seeking phase of the DFS algorithm proposed here. The validity of this point requires further research.

\subsection{Algorithmic details and numerical demonstration}

Our experiments were carried out using the public-domain software package SNARK14 [16]. In all experiments the initial vector $\overline{\boldsymbol{x}}$ was the 235,225 -dimensional zero vector (all components 0 ).

The relaxation parameter in Algorithm 3 was $\lambda=0.05$. Another issue that needs specification is the ordering of the constraints in (1), because the output of Algorithm 3 depends not only on the set of constraints, but also on their order. We used in our experiments the so-called efficient ordering, since it has been demonstrated to lead to better results faster when incorporated into ART [25, page 209].

In Algorithm 2, the number $N$ of perturbation steps (for each feasibility-seeking step) was 100,000 and we used $\gamma_{\ell}=b a^{\ell}$, with $b=0.02$ and $a=0.999,999$. The infinite sequence $\left(\mathbf{c}^{m}\right)_{m=0}^{\infty}$ was obtained by repetitions of the length- $2 J$ subsequence $\left(\mathbf{e}^{1}, \mathbf{e}^{2}, \ldots \mathbf{e}^{J},-\mathbf{e}^{1},-\mathbf{e}^{2}, \ldots,-\mathbf{e}^{J}\right)$. 


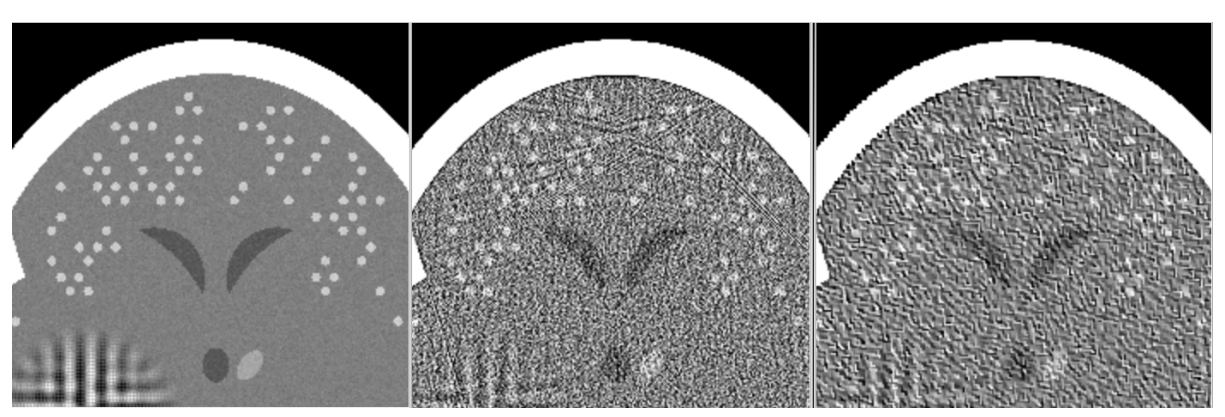

Fig. 1 Detail of (left) a $485 \times 485$ digitization of a phantom based on the distribution of x-ray attenuation in units of $\mathrm{cm}^{-1}$ within a transaxial slice of the human head (center) reconstruction produced by Algorithm 2 without its component-wise perturbations steps, and (right) produced by Algorithm 2 with its component-wise perturbations steps. In all images we display a value that is 0.20 or less as black and a value that is 0.22 or greater as white.

We applied Algorithm 2 twice, thirty iterations in each case, with and without its component-wise perturbations steps, respectively, under otherwise completely identical conditions; in Figure 1 we show a detail of the results produced by these two executions together with the corresponding detail of the digitized phantom. The resulting finite sequences of iterates are both of monotone proximity, the associated proximity-target curves are shown in Figure 2. The os and $*_{s}$ on the plots represent actually calculated values at iterations of each algorithm, that are connected by line segments. For any proximity value on the horizontal axis we can read the target-function value associated with it from the curve. The plots indicate visually the behavior of the algorithms, initialized at the same point denoted by $x^{0}=y^{0}$ that appears in the right-most side of the figure. The V-shaped form of the proximity-target curve for Algorithm 2 with perturbations is typical for the behavior of superiorized feasibility-seeking algorithms, showing the initially strong effect of the perturbations that diminishes as the iterations proceed.

For this experiment we used SNARK14 [16] installed in a computer with a 2.7 $\mathrm{GHz}$ Intel ${ }^{\circledR}$ Core 64 -bit i7 processor and a total main memory of 16 Gbytes running the CentOS Linux operating system on a virtual machine with 8 Gbytes assigned for base memory. Under these conditions, the implementation of Algorithm 2 without component-wise perturbations steps took 77.251 seconds to execute all the iterations whereas the implementation with such perturbations took 105.801 seconds for all the iterations. The latter time was measured excluding the time the implementation took to compute the useless Steps 12 to 18 for the first iteration (i.e., $k=0$ ). These steps are useless in our case, since with the zero vector as initial value, the condition in Step 16 is never satisfied while $k=0$, resulting in $\boldsymbol{x}^{0, N}=\boldsymbol{x}^{0}$.

For a more precise interpretation, consider Definition 7. In the experiment evaluating the two versions of Algorithm 2, $K_{l o}=L_{l o}=1$ and $K_{h i}=L_{h i}=$ 30. The $R=\left(\boldsymbol{x}^{k}\right)_{k=K_{l o}}^{K_{h i}}$ and $S=\left(\boldsymbol{y}^{k}\right)_{k=L_{l o}}^{L_{h i}}$ produced by Algorithm 2, with and without perturbations, respectively, are both of monotone proximity. We find that $\mathcal{P r}_{T}\left(\boldsymbol{x}^{K_{l o}}\right)=\operatorname{Pr}_{T}\left(\boldsymbol{y}^{L_{l o}}\right)=35.4703$ (and, hence, $u=35.4703$ ) and that $\mathcal{P} r_{T}\left(\boldsymbol{x}^{K_{h i}}\right)=3.4065$ and $\mathcal{P} r_{T}\left(\boldsymbol{y}^{L_{h i}}\right)=4.7828$ (and, hence, $t=4.7828$ ). By showing the target curves $P$ and $Q$ associated with $R$ and $S$, respectively, Figure 2 clearly illustrates that $R$ is better targeted than $S$. 


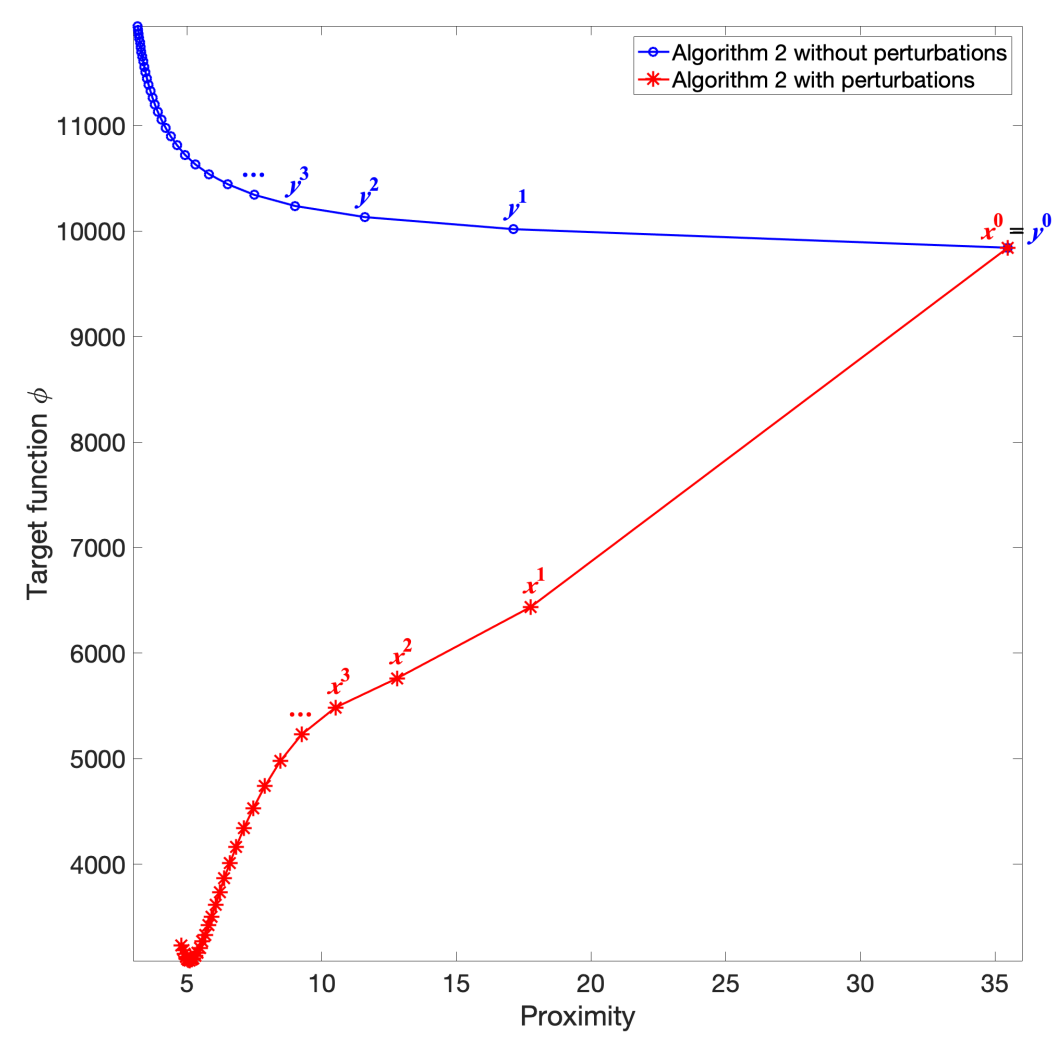

Fig. 2 Proximity-target curves $P$ and $Q$ of the first 30 iterates of Algorithm 2 with perturbations (*) and without perturbations (o).

\section{Discussion and conclusions}

In this paper we investigated the general applicability of derivative-free superiorization (DFS) as an alternative to previously proposed superiorization approaches. In our computational demonstration, we generated the constraints and chose the target function from the application area of image reconstruction from projections in computerized tomography (CT). However, we use the demonstration for indicating only the numerical behavior of the algorithms. We do not investigate or comment on the potential usefulness of the resulting reconstructions in $\mathrm{CT}$, since that usefulness depends not so much on the numerical behavior of the algorithms as on the appropriateness of the modeling used to turn a physical problem into a mathematical one (for example, by the specific choice of target function). The numerical results of our demonstration attest, as seen from the proximity-target curves, to the mathematical efficacy of our derivative-free superiorization algorithm, but say nothing about its efficacy for providing an answer to a practical image reconstruction problem. (Nevertheless, we have observed while doing our experiment that, even from the image reconstruction quality point of view, DFS 
seems to be advantageous. For example, if we consider the distances between the phantom and the reconstructions -defined as the 2-norm between the representing vectors-, the smallest distance that we get as we iterate without perturbations is 0.0922 , while with the DFS perturbations it is 0.0863.)

Much of the literature on derivative-free minimization is concerned with unconstrained minimization or at most with bound-constraints on the variables, and many, if not all, proposed methods can handle only small-size problems efficiently. In contrast, the DFS method proposed here can handle any type of constraints for which a separate efficient derivative-free constraints-compatibility-seeking algorithm is available. Since the constraints-compatibility-seeking algorithm forms part of the proposed DFS method, the method can use exterior initialization (i.e., initializing the iterations at any point in space). Furthermore, and very importantly, very large-size problems can be accommodated.

Acknowledgements We thank Nikolaos Sahinidis and Katya Scheinberg for several informative mail exchanges that helped us see better the general picture. We are grateful to Sébastien Le Digabel for calling our attention to the work of Charles Audet and coworkers, particularly the Audet and Dennis paper [1] and the book of Audet and Hare [2]. We greatly appreciate the constructive referee report that helped us improve the paper.

\section{Conflict of interest}

The authors declare that they have no conflict of interest.

\section{References}

1. Audet, C. and Dennis J.E. JR., 2009. A progressive barrier for derivative-free nonlinear programming, SIAM Journal on Optimization, 20, 445-472.

2. Audet, C. and Hare, W., 2017. Derivative-Free and Blackbox Optimization, Springer International Publishing, Cham, Switzerland.

3. Bargetz, C., Reich, S., and Zalas, R., 2018. Convergence properties of dynamic stringaveraging projection methods in the presence of perturbations, Numerical Algorithms, 77, 185-209.

4. Butnariu, D., Reich, S., and Zaslavski, A. J., 2006. Convergence to fixed points of inexact orbits of Bregman-monotone and of nonexpansive operators in Banach spaces, in: Proceedings of Fixed Point Theory and its Applications, Mexico, Yokohama, 11-32.

5. Cegielski, A., 2012. Iterative Methods for Fixed Point Problems in Hilbert Spaces, SpringerVerlag.

6. Cegielski, A. and Al-Musallam, F., 2017. Superiorization with level control, Inverse Problems, 33, 044009.

7. Censor, Y., 2015. Weak and strong superiorization: Between feasibility-seeking and minimization, Analele Stiintifice ale Universitatii Ovidius Constanta-Seria Matematica, 23, 41-54.

8. Censor, Y., 2019. Superiorization and Perturbation Resilience of Algorithms: A Bibliography compiled and continuously updated, http://math.haifa.ac.il/yair/bib-superiorizationcensor.html, last updated: September 19, 2020.

9. Censor, Y. and Cegielski, A., 2015. Projection methods: An annotated bibliography of books and reviews, Optimization, 64, 2343-2358.

10. Censor, Y., Heaton, H., and Schulte, R.W., 2019. Derivative-free superiorization with component-wise perturbations, Numerical Algorithms, 80, 1219-1240.

11. Censor, Y., Herman, G.T., and Jiang, M., (Editors) 2017. Special issue on Superiorization: Theory and Applications, Inverse Problems, 33, 040301-044014. 
12. Censor, Y., Levy, E., 2019. An analysis of the superiorization method via the principle of concentration of measure, Applied Mathematics and Optimization. https://doi.org/10.1007/s00245-019-09628-4.

13. Censor, Y. and Zaslavski, A., 2015. Strict Fejér monotonicity by superiorization of feasibility-seeking projection methods, Journal of Optimization Theory and Applications, $165,172-187$.

14. Conn, A.R., Scheinberg, K., and Vicente, L.N., 2009. Introduction to Derivative-Free Optimization, Society for Industrial and Applied Mathematics (SIAM).

15. Cooley, T.A. and Barrett, H.H., 1992. Evaluation of statistical methods for image reconstruction through ROC analysis, IEEE Transactions on Medical Imaging, 11, 276-282.

16. Davidi, R., Garduño, E., Herman, G.T., Langthaler, O., Rowland, S.W., Sardana, S., and Ye, Z., 2019. SNARK14: A programming system for the reconstruction of 2D images from 1D projections, available from http://turing.iimas.unam.mx/SNARK14M/SNARK14.pdf.

17. Diniz-Ehrhardt, M., Martínez, J., and Pedroso, L., 2011. Derivative-free methods for nonlinear programming with general lower-level constraints, Computational and Applied Mathematics, 30, 19-52.

18. Echeverría Ciaurri, D., Isebor, O., and Durlofsky, L., 2012. Application of derivative-free methodologies to generally constrained oil production optimization problems, Procedia Computer Science, 1, 1301-1310.

19. Engl, H.W., Hanke, M., and Neubauer, A., 2000. Regularization of Inverse Problems, Kluwer Academic Publishers.

20. Fletcher, R. and Leyffer, S., 2002. Nonlinear programming without a penalty function, Mathematical Programming, Series A, 91, 239-269.

21. Garduño, E. and Herman, G.T., 2014. Superiorization of the ML-EM algorithm, IEEE Transactions on Nuclear Science, 61, 162-172.

22. Garduño, E. and Herman, G.T., 2017. Computerized tomography with total variation and with shearlets, Inverse Problems, 33, 044011.

23. Gay, H.A., Niemierko, A., 2007. A free program for calculating EUD-based NTCP and TCP in external beam radiotherapy, Physica Medica, 23, 115-25.

24. He, H. and $\mathrm{Xu}$, H.K., 2017. Perturbation resilience and superiorization methodology of averaged mappings, Inverse Problems, 33, 044007.

25. Herman, G.T., 2009. Fundamentals of Computerized Tomography: Image Reconstruction from Projections, Springer-Verlag, 2nd ed.

26. Herman, G.T., 2019. Iterative reconstruction techniques and their superiorization for the inversion of the Radon transform, in: R. Ramlau and O. Scherzer, eds., The Radon Transform: The First 100 Years and Beyond, De Gruyter, 217-238.

27. Herman, G.T., 2020. Problem structures in the theory and practice of superiorization, Journal of Applied and Numerical Optimization, 2, 71-76.

28. Herman, G.T., Garduño, E., Davidi, R., and Censor, Y., 2012. Superiorization: An optimization heuristic for medical physics, Medical Physics, 39, 5532-5546.

29. Hoseini, M., Saeidi, S. and Kim, D.S., 2019. On perturbed hybrid steepest descent method with minimization or superiorization for subdifferentiable functions. Numerical Algorithms. https://doi.org/10.1007/s11075-019-00818-3.

30. Kaczmarz, S., 1937. Angenäherte Auflösung von Systemen linearer Gleichungen, Bulletin de l'Académie Polonaise des Sciences et Lettres, A35, 355-357.

31. Kolda, T., R. Lewis and V. Torczon, 2003. Optimization by direct search: New perspectives on some classical and modern methods, SIAM Review, 45, 385-482.

32. Li, L., Chen, Y., Liu, Q., Lazic, J., Luo, W., and Li, Y., 2017. Benchmarking and evaluating MATLAB derivative-free optimisers for single-objective applications, in: D.S. Huang, K.H. Jo, and J. Figueroa-García, eds., Intelligent Computing Theories and Application. ICIC 2017, Springer, 75-88.

33. Luo, S., Zhang, Y., Zhou, T., and Song, J., 2016. Superiorized iteration based on proximal point method and its application to XCT image reconstruction, ArXiv e-prints, https://arxiv.org/abs/1608.03931.

34. Luo, S., Zhang, Y., Zhou, T., Song, J., and Wang, Y., 2019. XCT image reconstruction by a modified superiorized iteration and theoretical analysis, Optimization Methods and Software, DOI: 10.1080/10556788.2018.1560442.

35. Metz, C.E., Some practical issues of experimental design and data analysis in radiological ROC studies, Investigative Radiology, 24, 234-243.

36. Nikazad, T., Davidi, R., and Herman, G.T., 2012. Accelerated perturbation resilient blockiterative projection methods with application to image reconstruction, Inverse Problems, $28,035005$. 
37. Nystrom, H., Jensen, M.F., Nystrom, P.W., 2020. Treatment planning for proton therapy: what is needed in the next 10 years? The British Journal of Radiology, 93, 1107, 20190304. DOI:10.1259/bjr.20190304.

38. Reich, S. and Zalas, R., 2016. A modular string averaging procedure for solving the common fixed point problem for quasi-nonexpansive mappings in Hilbert space, Numerical Algorithms, 72, 297-323.

39. Reich, S. and Zaslavski, A.J., 2017. Convergence to approximate solutions and perturbation resilience of iterative algorithms, Inverse Problems, 33, 044005.

40. Rios, L.M. and Sahinidis, N.V., 2013. Derivative-free optimization: A review of algorithms and comparison of software implementations, Journal of Global Optimization, 56, $1247-1293$.

41. Swets, J.A.,1979. ROC analysis applied to the evaluation of medical imaging techniques, Investigative Radiology, 14, 109-112.

42. Zaslavski, A.J., 2018. Algorithms for Solving Common Fixed Point Problems, Springer International Publishing. 